\title{
Dor lombar crônica em uma população de mulheres do Sul do Brasil: prevalência e fatores associados
}

\author{
Chronic low back pain in a population of women in Southern Brazil: \\ prevalence and associated factors \\ Lumbalgia crónica en mujeres de la región Sur de Brasil: prevalencia y factores asociados \\ Patrícia Cilene Freitas Sant'Anna', Maria Teresa Anselmo Olinto², Fernanda Souza de Bairros³, \\ Anderson Garcez ${ }^{4}$, Juvenal Soares Dias da Costa ${ }^{5}$
}

RESUMO I O objetivo deste estudo foi identificar a prevalência de dor lombar crônica e seus fatores associados em uma população de mulheres. Para isso, realizou-se um estudo transversal, de base populacional, com 1.128 mulheres com idade entre 20 e 69 anos e residentes na zona urbana de São Leopoldo, Rio Grande do Sul. A dor lombar crônica foi avaliada a partir da presença de dor com duração mínima de três meses, e os fatores investigados incluíram aspectos sociodemográficos, comportamentais e antropométricos. Os resultados indicaram uma prevalência de dor lombar crônica de 46,5\% (IC95\%: 43,6-49,4). Após ajuste, os fatores associados à dor lombar crônica foram: idade, atividade física e estado nutricional. Observou-se uma associação com tendência linear positiva entre idade e dor lombar, assim como uma probabilidade 31\% (RP=1,31; IC95\%: 1,051,64; $p=0,02$ ) e 25\% ( $R P=1,25 ;$ IC95\%: 1,09-1,42; $p<0,001)$ maior de mulheres fisicamente inativas e obesas terem dor lombar, quando comparadas às mulheres fisicamente ativas e com peso corporal normal, respectivamente. Assim, este estudo evidenciou uma elevada prevalência de dor lombar crônica em mulheres, identificando como principais fatores associados a sua ocorrência a idade avançada, a inatividade física e a obesidade.
Descritores | Dor Lombar; Mulheres; Estudo Transversal; Epidemiologia.

ABSTRACT I This study sought to identify the prevalence of chronic low back pain and its associated factors in a population of women. A cross-sectional populationbased study was conducted with 1,128 women aged 20 to 69 and living in the urban area of São Leopoldo, Rio Grande do Sul (Brazil). Chronic low back pain was assessed by the presence of prevailing pain lasting for at least three months, and the investigated factors consisted of sociodemographic, behavioral and anthropometric aspects. The prevalence of chronic low back pain was 46.5\% (95\% Cl: 43.6-49.4). After the adjustment, factors associated with the presence of chronic low back pain were: age, physical activity and nutritional status. We observed an association with a positive linear trend between age and low back pain. Furthermore, physically inactive and obese women presented, respectively, a 31\% (PR=1.31; 95\% Cl: 1.05-1.64; $p=0.02)$ and $25 \%(P R=1.25 ; 95 \% C l: 1.09-1.42 ; p<0.001)$ higher probability of presenting low back pain than those with normal body weight. In conclusion, we identified a high prevalence of chronic low back pain in women, and

\footnotetext{
Estudo realizado na Universidade do Vale do Rio dos Sinos (Unisinos).

Universidade do Vale do Rio dos Sinos (Unisinos) - São Leopoldo (RS), Brasil. E-mail: pcfsantanna@gmail.com. Orcid: 0000-0002-8278-8692

²Universidade do Vale do Rio dos Sinos (Unisinos) - São Leopoldo (RS), Brasil. E-mail: mtolinto@gmail.com. Orcid: 0000-0002-3950-4594

3Universidade Federal do Rio Grande do Sul (UFRGS) - Porto Alegre (RS), Brasil. E-mail: fernandabairros@gmail.com. Orcid: 0000-0002-8611-058X

4Universidade do Vale do Rio dos Sinos (Unisinos) - São Leopoldo (RS), Brasil - Porto Alegre (RS), Brasil. E-mail: adsgarcez@gmail.com. Orcid: 0000-0003-1111-4890

Unniversidade do Vale do Rio dos Sinos (Unisinos) - São Leopoldo (RS), Brasil. E-mail: episoares@terra.com.br.

Orcid: 0000-0003-3160-6075
} 
the main associated factors included: advanced age, physical inactivity and obesity.

Keywords I Low Back Pain; Women; Cross-Sectional Studies; Epidemiology.

RESUMEN | El objetivo de este estudio fue identificar la prevalencia de lumbalgia crónica y sus factores asociados en mujeres. Se realizó un estudio transversal, poblacional, con 1.128 mujeres de entre 20 y 69 años de edad, residentes en la zona urbana de São Leopoldo, en el estado de Rio Grande do Sul (Brasil). Para caracterizar lumbalgia crónica se evaluó la presencia de dolor durante al menos tres meses, y los factores investigados incluyeron aspectos sociodemográficos, conductuales y antropométricos. Los resultados apuntan una prevalencia de lumbalgia crónica del 46,5\% (IC95\%: 43,6-49,4). Tras el ajuste, los factores asociados a la lumbalgia crónica fueron: edad, actividad física y estado nutricional. Hubo una asociación de tendencia lineal positiva entre la edad y la lumbalgia, así como una probabilidad del 31\% (RP=1,31; IC95\%: 1,05-1,64; $p=0,02$ ) y del 25\% (RP= 1,25; IC95\%: 1,09-1,42; $p<0,001$ ) mayor de dolor lumbar en mujeres físicamente inactivas y obesas en comparación con mujeres físicamente activas y con peso corporal normal. Este estudio evidenció una alta prevalencia de lumbalgia crónica en mujeres y los principales factores asociados fueron la edad avanzada, la inactividad física y la obesidad.

Palabras clave | Dolor Lumbar; Mujeres; Estudio Transversal; Epidemiología.

\section{INTRODUÇÃO}

A dor lombar é considerada um dos maiores problemas mundiais de saúde, posto que aproximadamente $12 \%$ das pessoas com esse distúrbio relatam limitação por mais de um $\operatorname{dia}^{1}$. Suas consequências podem afetar o trabalho, a vida social e o lazer, assim como gerar gastos diretos para o indivíduo e para o Estado ${ }^{2}$. Além disso, a dor lombar está entre as mais comuns complicações de saúde que conduzem as pessoas a consultas médicas, sendo classificada como uma importante causa de incapacidade funcional ${ }^{3}$. Contudo, frequentemente essa enfermidade é vista como um problema de saúde trivial quando comparada com outras afecções de alta mortalidade ${ }^{4}$.

A dor lombar pode ser caracterizada como crônica quando persistir por três meses ou mais ${ }^{5}$. Nos Estados Unidos, o custo com a dor lombar crônica tem sido cada vez maior. Algumas evidências sugerem que isso decorre do possível aumento de seu nível de cronicidade ${ }^{6}$. Ademais, dados apontam um crescimento na ocorrência de dor lombar crônica, que passou de 3,9\% para 10,2\% em um período de 14 anos $^{7}$. No Brasil, estudos indicam que as doenças crônicas de coluna são relevantes causas de morbidade entre adultos e idosos ${ }^{8,9}$. Nesse sentido, dados da Pesquisa Nacional de Saúde de 2013 revelaram que, entre as afecções crônicas, as doenças de coluna ocupavam o segundo lugar em termos de prevalência, acometendo aproximadamente $18,5 \%$ da população adulta ${ }^{9}$. De forma similar, estudo conduzido no Sul do Brasil apontou aumento da prevalência de dor lombar crônica, que passou de 4,2\% para 9,6\% em um período de oito anos ${ }^{10}$.
A dor lombar, ainda que seja uma manifestação de caráter físico, não está relacionada somente a aspectos fisiológicos, como revelam alguns estudos que associam sua ocorrência a outros determinantes, incluindo aspectos demográficos ${ }^{11-14}$, socioeconômicos ${ }^{14,15}$, comportamentais $^{13,16}$ e antropométricos ${ }^{12,15,17}$. Dessa forma, diversas investigações de base populacional têm verificado que a dor lombar crônica é significativamente maior entre as mulheres do que entre os homens ${ }^{7,12-14,18}$. Diante disso, o objetivo principal deste estudo foi identificar a prevalência de dor lombar crônica e os fatores a ela associados em uma população de mulheres do Sul do Brasil. Considera-se que pesquisas de base populacional sobre a prevalência de dor lombar crônica e os fatores a ela associados são importantes por quantificarem a magnitude do problema e, com isso, poderem direcionar programas e ações preventivas contra a ocorrência de dor lombar, principalmente em grupos populacionais vulneráveis.

\section{METODOLOGIAS}

Realizou-se um estudo epidemiológico transversal de base populacional, incluindo uma amostra representativa de mulheres com idade entre 20 e 69 anos e residentes na área urbana da cidade de São Leopoldo, Rio Grande do Sul. Este estudo integrou um projeto de pesquisa maior, destinado a avaliar as condições de vida e saúde de mulheres de um município localizado na região do Vale do Rio dos Sinos, Rio Grande do Sul. 
O tamanho da amostra do estudo foi calculado com base em diversos desfechos em saúde, escolhendo-se o que exigia maior amostragem (realização de exame citopatológico). Os pressupostos para o cálculo foram: razão de risco de 2,0, nível de confiança de $95 \%$, poder estatístico de $80 \%$ e razão de não expostos:expostos de 1:2 para a variável escolaridade. Acrescentou-se uma taxa de $10 \%$ para possíveis perdas/recusas e de $15 \%$ para controle de fatores de confusão, resultando em 1.281 mulheres. Em 2010, a população da cidade de São Leopoldo era de 217.189 pessoas, das quais 111.435 eram mulheres (51,3\%). Destas, 72.775 tinham idade entre 20 e 69 anos, correspondendo a 65,3\% da população feminina.

Com base na média de pessoas por domicílio $(2,99)$, na proporção de mulheres na faixa etária de interesse $(32,1 \%)$, segundo o Censo Demográfico 2010 do Instituto Brasileiro de Geografia e Estatística $(\mathrm{IBGE})^{19}$, e no tamanho necessário da amostra (1.281), estimou-se um total de 1.335 domicílios a serem visitados. A amostragem ocorreu por conglomerados, em dois estágios. No primeiro, selecionaram-se os setores censitários e, no segundo, os domicílios. Para garantir maior representatividade, foram escolhidos de maneira sistemática 45 dos 371 setores existentes em São Leopoldo e, em cada setor, 36 domicílios. Os critérios de inclusão no estudo foram: ter de 20 a 69 anos e residir no domicílio sorteado. Os critérios de exclusão, por sua vez, foram: ser gestante ou apresentar limitações físicas e/ou mentais que impossibilitassem obter os dados.

As características da amostra foram obtidas em visitas domiciliares, por entrevistadores treinados, entre fevereiro e outubro de 2015, por meio da aplicação de questionários padronizados, pré-codificados e pré-testados. Foi realizado um estudo-piloto em um setor censitário da zona urbana de São Leopoldo, não incluído entre os sorteados para o estudo efetivo, cujo objetivo principal era treinar os entrevistadores, fazer o teste final dos instrumentos, aprimorar os processos de codificação, a entrada de dados e a realização de análise inicial. O estudo contou com supervisores de trabalho de campo, que semanalmente empreendiam reuniões com os entrevistadores para entregar os questionários e verificar o andamento do trabalho de campo. No caso de recusa dos sorteados, realizavam-se mais duas tentativas em dias e horários diferentes. Se o insucesso continuasse, uma última tentativa era efetuada por um dos supervisores. Ademais, procedeu-se a um controle de qualidade em 10\% da amostra estudada, mediante aplicação de um questionário com 10 questões do instrumento original, incluindo variáveis que não sofriam alteração em curto período.

A ocorrência de dor lombar crônica foi avaliada com base na presença de dor ou desconforto na região entre a última costela e o final do glúteo ${ }^{20}$, com duração mínima de três meses ${ }^{17}$. Para adequada identificação da região lombar, foi utilizado um cartão-resposta contendo uma ilustração corporal adaptada, com destaque para a região lombar ${ }^{21}$. Por sua vez, as variáveis independentes investigadas e exploradas, que se relacionavam à ocorrência de dor lombar crônica, contemplaram características sociodemográficas, comportamentais e nutricionais (medida antropométrica). Adicionalmente, uma possível presença de incômodo ou limitação para realizar atividades da vida diária em decorrência da dor lombar foi explorada por meio de questões sobre a dificuldade em executar tarefas básicas nas duas últimas semanas, como se vestir ou caminhar longas distâncias.

As variáveis sociodemográficas deste estudo foram: idade (20-29, 30-39, 40-49, 50-59 e 60-69); cor da pele (branca e não branca); situação conjugal (solteira, casada, separada e viúva); nível de escolaridade ( $\geq 15,11$ a 14,8 a 10, 5 a 7 e $\leq 4$ anos de estudo); renda familiar per capita expressa em número de salários mínimos ( $>3$ salários mínimos, de 1 a 3 salários mínimos e $<1$ salário mínimo); e classe econômica, definida de acordo com os Critérios de Classificação Econômica 2011 da Associação Brasileira de Empresas de Pesquisa (Abep) (classes A-E). As variáveis comportamentais, por sua vez, foram: atividade física fora da atuação laboral, medida com base no Questionário Internacional de Atividade Física (Ipaq), versão curta e adaptada (fisicamente ativa - com no mínimo 75 minutos semanais de atividades intensas ou 150 minutos semanais de atividades moderadas -, e fisicamente inativa); comportamento sedentário, expresso em número de horas na posição sentada durante o dia ( $<4$ horas, de 4 a $<6$ horas, de 6 a 8 horas e $>8$ horas); e tabagismo (não fumante, ex-fumante há mais de 10 anos, ex-fumante há 10 anos ou menos e fumante). Por sua vez, o estado nutricional foi avaliado por meio do índice de massa corporal (IMC), obtido pelo peso/ altura $^{2}$ e classificado em normal $\left(<25 \mathrm{~kg} / \mathrm{m}^{2}\right)$, sobrepeso $\left(\right.$ IMC $\geq 25$ e $\left.<30 \mathrm{~kg} / \mathrm{m}^{2}\right)$ e obesidade $\left(\geq 30 \mathrm{~kg} / \mathrm{m}^{2}\right)$. Para coletar as medidas e calcular o peso e a altura corporal foram utilizadas balanças portáteis e antropômetros fixos. Para verificar as medidas, solicitou-se às entrevistadas 
que ficassem descalças e com a menor quantidade de roupa possível.

Os dados foram inseridos no programa EpiData, versão 3.1, com dupla digitação, a fim de corrigir eventuais erros. Procedeu-se então à análise estatística nos Programas SPSS, versão 22, e Stata, versão 12, de forma que as características investigadas foram descritas por meio de frequências absolutas e relativas. A associação entre o desfecho e as variáveis independentes exploradas utilizou os testes de qui-quadrado e de tendência linear para as variáveis categóricas e ordinais, respectivamente. As razões de prevalência $(\mathrm{RP})$ brutas e ajustadas para as associações, com intervalos de confiança de 95\% (IC 95\%), foram obtidas mediante regressão de Poisson com variância robusta, contemplando um modelo de regressão ajustado e hierarquizado em três níveis ${ }^{22}$. O primeiro nível, mais distal, incluiu as variáveis demográficas e socioeconômicas, que poderiam ser fatores determinantes das variáveis comportamentais do segundo nível. No terceiro nível incluiu-se a variável de estado nutricional. Foram selecionadas e incluídas na análise ajustada apenas as variáveis que obtiveram $\mathrm{p}$-valor menor ou igual a $10 \%(\mathrm{p} \leq 0,10)$ na análise bruta, permanecendo no modelo final somente aquelas com significância estatística menor do que $5 \%(\mathrm{p}<0,05)$. Por se tratar de estudo com amostragem por conglomerados, utilizou-se o comando "svy" do Stata, considerando um efeito do delineamento de 1,08.
Ademais, calcularam-se as frações atribuíveis para os fatores de risco modificáveis entre a população (fatores relacionados aos hábitos de vida, como tabagismo, sedentarismo e obesidade), ou seja, a proporção de prevalência de dor lombar crônica que poderia ser diminuída caso cessasse a exposição a esses fatores. Para calcular a fração atribuível empregou-se a fórmula $\mathrm{P}_{\mathrm{e}}(\mathrm{RP}-1) / 1+\mathrm{P}_{\mathrm{e}}$ $(\mathrm{RP}-1)$, sendo $\mathrm{P}_{\mathrm{e}}$ a proporção de exposição na população e $\mathrm{RP}$ a razão de prevalência (prevalência da doença entre os expostos/prevalência da doença entre os não expostos).

\section{RESULTADOS}

Um total de 1.128 mulheres foram entrevistadas e incluídas na análise final deste estudo. Entre as características gerais da amostra, observaram-se um maior percentual de mulheres adultas na faixa etária entre 40 e 49 anos $(24,5 \%)$ e um menor percentual de mulheres idosas com 60 anos ou mais de idade (14,5\%). A maioria das entrevistadas era branca $(74,5 \%)$, casada/ em união $(63,8 \%)$, com escolaridade entre 11 e 14 anos de estudo (32\%), tinha renda familiar inferior a um salário mínimo $(60,8 \%)$, pertencia à classe econômica $\mathrm{C}(53,1 \%)$, era fisicamente inativa $(85,6 \%)$, permanecia menos de quatro horas por dia sentada $(43,1 \%)$, não apresentava histórico de tabagismo $(59,3 \%)$ e era classificada com excesso de peso corporal $(66,1 \%)$ (Tabela 1$)$.

Tabela 1. Características gerais da amostra e distribuição da prevalência de dor lombar crônica de acordo com características sociodemográficas, comportamentais e de estado nutricional em mulheres do Sul do Brasil ( $n=1128)$

\begin{tabular}{|c|c|c|c|}
\hline Características & n (\%) & Prevalência de dor lombar (\%) & p-valor \\
\hline Idade (anos) & & & $<0,001^{*}$ \\
\hline 20 a 29 & $216(19,1)$ & $68(31,5)$ & \\
\hline 30 a 39 & $244(21,6)$ & $103(42,2)$ & \\
\hline 40 a 49 & $276(24,5)$ & $147(53,3)$ & \\
\hline 50 a 59 & $228(20,2)$ & $122(53,5)$ & \\
\hline 60 a 69 & $164(14,5)$ & $85(51,8)$ & \\
\hline Cor da pele & & & $0,730^{* *}$ \\
\hline Branca & $840(74,5)$ & $388(46,2)$ & \\
\hline Não branca & $288(25,5)$ & $137(47,6)$ & \\
\hline \multicolumn{4}{|l|}{ Situação conjugal } \\
\hline Solteira & $227(20,1)$ & $83(36,6)$ & $0,001^{* *}$ \\
\hline Casada & $720(63,8)$ & $347(48,2)$ & \\
\hline Separada & $110(9,8)$ & $65(59,1)$ & \\
\hline Viúva & $71(6,3)$ & $30(42,3)$ & \\
\hline
\end{tabular}


Tabela 1. Continuação

\begin{tabular}{|c|c|c|c|}
\hline Características & $n(\%)$ & Prevalência de dor lombar (\%) & p-valor \\
\hline Escolaridade (anos) ( $n=1126)$ & & & $<0,001^{*}$ \\
\hline$\geq 15$ & $110(9,8)$ & $36(32,7)$ & \\
\hline 11 a 14 & $360(32,0)$ & $148(41,1)$ & \\
\hline 8 a 10 & $199(17,7)$ & $102(51,3)$ & \\
\hline 5 a 7 & $253(22,5)$ & $129(51,0)$ & \\
\hline$\leq 4$ & $204(18,1)$ & $109(53,4)$ & \\
\hline Renda familiar (SM) $(n=1091)$ & & & $0,030^{*}$ \\
\hline$>3$ & $66(6,0)$ & $23(34,8)$ & \\
\hline $1-3$ & $362(33,2)$ & $163(45,0)$ & \\
\hline$<1$ & $663(60,8)$ & $324(48,9)$ & \\
\hline Classificação econômica (n=1122) & & & $0,070^{*}$ \\
\hline A & $44(3,9)$ & $14(31,8)$ & \\
\hline B & $346(30,8)$ & $149(43,1)$ & \\
\hline C & $596(53,1)$ & $300(50,3)$ & \\
\hline$D / E$ & $136(12,2)$ & $60(44,1)$ & \\
\hline Atividade física & & & $0,010^{* *}$ \\
\hline Fisicamente ativa & $162(14,4)$ & $59(36,4)$ & \\
\hline Fisicamente inativa & $966(85,6)$ & $466(48,2)$ & \\
\hline \multicolumn{4}{|l|}{ Comportamento sedentário (horas) } \\
\hline$<4$ & $486(43,1)$ & $239(49,2)$ & $0,200^{*}$ \\
\hline $4 \mathrm{a}<6$ & $196(17,4)$ & $100(51,0)$ & \\
\hline 6 a 8 & $166(14,7)$ & $70(42,2)$ & \\
\hline$>8$ & $280(24,8)$ & $116(41,4)$ & \\
\hline \multicolumn{4}{|l|}{ Tabagismo $(n=1115)$} \\
\hline Nunca fumou & $661(59,3)$ & $293(44,3)$ & $0,150^{* *}$ \\
\hline Ex-fumante (>10 anos) & $131(11,7)$ & $63(48,1)$ & \\
\hline Ex-fumante ( $\leq 10$ anos) & $107(9,6)$ & $59(55,1)$ & \\
\hline Fumante & $216(19,4)$ & $107(49,5)$ & \\
\hline \multicolumn{4}{|l|}{ Estado nutricional $(\mathrm{n}=1122)$} \\
\hline Normal $\left(I M C<25 \mathrm{~kg} / \mathrm{m}^{2}\right)$ & $380(33,9)$ & $153(40,3)$ & \\
\hline Sobrepeso $\left(25 \leq 1 M C<30 \mathrm{~kg} / \mathrm{m}^{2}\right)$ & $373(33,2)$ & $164(44,0)$ & $<0,001^{*}$ \\
\hline Obesidade (IMC $\left.\geq 30 \mathrm{~kg} / \mathrm{m}^{2}\right)$ & $369(32,9)$ & $206(55,8)$ & \\
\hline
\end{tabular}

SM: Salários mínimos; IMC: Índice de massa corporal; 'Teste qui-quadrado de Pearson para tendência linear; "Teste qui-quadrado de Pearson para heterogeneidade de proporções.

A prevalência de dor lombar crônica entre as mulheres investigadas foi de 46,5\% (IC 95\%: 43,6-49,4) e 59\% das mulheres com dor lombar relataram algum grau de incômodo ou limitação nas atividades diárias nas duas últimas semanas, como evitar caminhar longas distâncias ou precisar se vestir mais devagar do que o habitual. $\mathrm{Na}$ análise bruta dos dados observou-se uma associação entre a dor lombar crônica e as variáveis sociodemográficas idade, estado civil, escolaridade e classe econômica (Tabela 1). Quanto às variáveis comportamentais, notouse uma associação significativa entre dor lombar crônica e atividade física, mas não foram encontradas diferenças significativas na relação entre dor lombar crônica, comportamento sedentário e tabagismo. Por outro lado, o estado nutricional apresentou associação significativa com a presença de dor lombar crônica (Tabela 1).
Após ajuste, os fatores que permaneceram estatisticamente associados à presença de dor lombar crônica foram: idade, atividade física e estado nutricional (Tabela 2). Observou-se associação com tendência linear positiva entre o aumento da idade e a ocorrência de dor lombar. Por sua vez, as mulheres fisicamente inativas apresentaram uma probabilidade $31 \%$ maior de ter dor lombar, quando comparadas àquelas fisicamente ativas $(\mathrm{RP}=1,31$; $\mathrm{IC}$ 95\%: 1,05-1,64; $\mathrm{p}=0,02)$, assim como as mulheres obesas tiveram $25 \%$ mais chances de apresentarem dor lombar ( $\mathrm{RP}=1,25$; IC 95\%: 1,09$1,42 ; \mathrm{p}<0,001)$ quando comparadas às mulheres com peso corporal normal (Tabela 2). Por fim, as frações atribuíveis aos fatores relacionados aos hábitos de vida foram de $22,5 \%$ para atividade física insuficiente e de $7,6 \%$ para obesidade. 
Tabela 2. Razões de prevalências (RP) brutas e ajustadas e seus respectivos intervalos de confiança (IC 95\%) para a associação entre dor lombar crônica de acordo com características sociodemográficas, comportamentais e de estado nutricional em mulheres do Sul do Brasil $(n=1128)$

\begin{tabular}{|c|c|c|c|}
\hline \multirow{2}{*}{ Características } & \multirow{2}{*}{$\begin{array}{l}\text { Análise bruta } \\
\text { RP (IC 95\%) }\end{array}$} & \multicolumn{2}{|c|}{ Análise ajustada } \\
\hline & & RP (IC 95\%) & p-valor \\
\hline Idade (anos) ${ }^{a}$ & & & $<0,001^{*}$ \\
\hline 20 a 29 & 1,00 (referência) & 1,00 (referência) & \\
\hline 30 a 39 & $1,34(1,05-1,71)$ & $1,37(1,05-1,78)$ & \\
\hline 40 a 49 & $1,69(1,35-2,12)$ & $1,68(1,30-2,17)$ & \\
\hline 50 a 59 & $1,70(1,35-2,14)$ & $1,69(1,28-2,22)$ & \\
\hline 60 a 69 & $1,65(1,29-2,11)$ & $1,59(1,19-2,13)$ & \\
\hline \multicolumn{4}{|l|}{ Cor da pele ${ }^{a}$} \\
\hline Branca & 1,00 (referência) & & \\
\hline Não branca & $1,03(0,89-1,19)$ & & \\
\hline Situação conjugala & & & $0,120^{* *}$ \\
\hline Solteira & 1,00 (referência) & 1,00 (referência) & \\
\hline Casada & $1,32(1,09-1,59)$ & $1,16(0,95-1,43)$ & \\
\hline Separada & $1,62(1,28-2,04)$ & $1,27(0,98-1,64)$ & \\
\hline Viúva & $1,16(0,84-1,59)$ & $0,92(0,63-1,33)$ & \\
\hline Escolaridade (anos) ${ }^{a}(n=1126)$ & & & $0,240^{*}$ \\
\hline$\geq 15$ & 1,00 (referência) & 1,00 (referência) & \\
\hline 11 a 14 & $1,26(0,94-1,69)$ & $0,95(0,79-1,14)$ & \\
\hline 8 a 10 & $1,57(1,16-2,11)$ & $1,05(0,85-1,29)$ & \\
\hline 5 a 7 & $1,56(1,16-2,09)$ & $0,89(0,71-1,12)$ & \\
\hline$\leq 4$ & $1,63(1,21-2,20)$ & $0,73(0,52-1,03)$ & \\
\hline Renda familiar $(S M)^{a}(n=1091)$ & & & $0,500^{*}$ \\
\hline$>3$ & 1,00 (referência) & 1,00 (referência) & \\
\hline $1-3$ & $1,29(0,91-1,83)$ & $1,14(0,78-1,67)$ & \\
\hline$<1$ & $1,40(1,00-1,97)$ & $1,20(0,82-1,76)$ & \\
\hline Classificação econômicaa ( $n=1122)$ & & & $0,420^{*}$ \\
\hline A & 1,00 (referência) & 1,00 (referência) & \\
\hline B & $1,35(0,86-2,12)$ & $1,22(0,82-1,82)$ & \\
\hline C & $1,58(1,02-2,46)$ & $1,28(0,84-1,95)$ & \\
\hline $\mathrm{D} / \mathrm{E}$ & $1,39(0,87-2,22)$ & $1,12(0,68-1,85)$ & \\
\hline Atividade físicab $^{\mathrm{b}}$ & & & $0,020^{* *}$ \\
\hline Fisicamente ativa & 1,00 (referência) & 1,00 (referência) & \\
\hline Fisicamente inativa & $1,32(1,07-1,64)$ & $1,31(1,05-1,64)$ & \\
\hline Comportamento sedentário (horas) & & & $0,560^{*}$ \\
\hline$<4$ & 1,00 (referência) & 1,00 (referência) & \\
\hline $4 a<6$ & $1,04(0,88-1,22)$ & $1,06(0,90-1,25)$ & \\
\hline 6 a 8 & $0,86(0,70-1,05)$ & $0,91(0,73-1,13)$ & \\
\hline$>8$ & $0,84(0,71-0,99)$ & $0,96(0,80-1,15)$ & \\
\hline \multicolumn{4}{|l|}{ Tabagismo $(n=1115)$} \\
\hline Nunca fumou & 1,00 (referência) & & \\
\hline Ex-fumante ( $>10$ anos) & $1,09(0,89-1,32)$ & & \\
\hline Ex-fumante ( $\leq 10$ anos) & $1,24(1,03-1,51)$ & & \\
\hline Fumante & $1,12(0,95-1,31)$ & & \\
\hline Estado nutricional $(n=1122)^{c}$ & & & $<0,001^{*}$ \\
\hline Normal $\left(I M C<25 \mathrm{~kg} / \mathrm{m}^{2}\right)$ & 1,00 (referência) & 1,00 (referência) & \\
\hline Sobrepeso $\left(25 \leq \mathrm{IMC}<30 \mathrm{~kg} / \mathrm{m}^{2}\right)$ & $1,09(0,92-1,29)$ & $1,00(0,86-1,16)$ & \\
\hline Obesidade $\left(\mathrm{IMC} \geq 30 \mathrm{~kg} / \mathrm{m}^{2}\right)$ & $1,39(1,19-1,62$ & $1,25(1,09-1,42)$ & \\
\hline
\end{tabular}

SM: Salários mínimos; IMC: Índice de massa corporal; *Teste Wald para tendência linear; **Teste Wald para heterogeneidade de proporções; ${ }^{\circledR}$ Variáveis ajustadas entre si; ${ }^{\circledR}$ Ajustada para idade, situação conjugal, escolaridade, renda familiar e classificação econômica; 'Ajustada para idade, situação conjugal, escolaridade, renda familiar, classificação econômica, atividade física e comportamento sedentário. 


\section{DISCUSSÃO}

Este estudo identificou alta prevalência de dor lombar crônica em um grupo de mulheres do Sul do Brasil, constatando como principais fatores a idade avançada, inatividade física e obesidade. A prevalência de dor lombar crônica na amostra investigada foi de 46,5\% (IC 95\%: 43,6-49,4), índice semelhante ao observado em estudos anteriores, a exemplo de pesquisa realizada na mesma cidade com 775 mulheres usuárias de um plano de saúde, que verificou prevalência de dor lombar crônica de 49,3\% (IC 95\%: 44,4-54,2) ${ }^{13}$. Por outro lado, estudos também verificaram prevalências menores para dor lombar crônica entre mulheres, incluindo pesquisas conduzidas em Pelotas, Rio Grande do Sul (11,7\%; IC 95\%: 10,0$13,4)^{18}$,em Salvador, Bahia (14,8\%; IC 95\%: 12,8-16,7) ${ }^{23}$, e em Portugal (14,1\%; IC 95\%: 12,7-15,3 $)^{12}$. Apesar de se caracterizarem como estudos transversais e de base populacional, as diferenças encontradas nas prevalências em relação a este estudo podem ser explicadas pelos tipos de questões utilizadas nas entrevistas e pelo recordatório dos participantes ${ }^{13,24}$.

Entre os resultados observados neste estudo, verificou-se que os principais fatores associados à dor lombar crônica entre as mulheres foram a idade avançada, inatividade física e obesidade. Nesse sentido, estudos prévios sobre o tema demonstraram associações significativas semelhantes entre dor lombar crônica e avanço da idade ${ }^{12-14}$, inatividade física $^{12,15,25}$ e obesidade ${ }^{12,14,15}$.

Em relação ao avanço da idade, a prevalência de dor lombar crônica tende a aumentar linearmente entre os 30 e os $60 \operatorname{anos}^{4,10}$. Essa associação tem uma plausibilidade biológica, pois, com o passar do tempo, o organismo humano inevitavelmente sofre um declínio em suas funções, levando a diversas alterações mecânicodegenerativas nas estruturas anatômicas ${ }^{16}$. Além disso, estudo de revisão sobre a temática verificou um pico da prevalência de dor lombar aos 60 anos de idade, com consequente diminuição dessa ocorrência em indivíduos acima de 60 anos $^{4}$. Segundo o estudo, muitos fatores poderiam explicar o declínio na prevalência que acontece a partir dos 60 anos, incluindo, por exemplo, possível comprometimento cognitivo, diminuição da percepção da dor ou maior tolerância à dor nessa fase da vida ${ }^{4}$. Contudo, em virtude do processo de envelhecimento populacional, o número de indivíduos com dor lombar tende a aumentar substancialmente nas próximas décadas ${ }^{1}$.

Quanto à prática de atividade física, observou-se que as mulheres fisicamente inativas apresentaram uma probabilidade $31 \%$ maior de ter dor lombar, em comparação às fisicamente ativas. Nesse sentido, um estudo sueco conduzido com 5.798 indivíduos com idade entre 25 e 79 anos apontou o baixo nível de atividade física no lazer como um dos principais fatores associados à dor lombar crônica ${ }^{15}$. Resultado semelhante foi identificado por um levantamento realizado em Portugal com 10.661 adultos, que verificou associação inversa entre a prática de atividade física e a dor lombar crônica ${ }^{12}$. Entretanto, existem pesquisas na literatura que não encontraram uma associação significativa entre dor lombar e prática de atividade física ${ }^{14,26}$. Além disso, há uma possível associação em forma de "U" entre nível de atividade física e dor lombar crônica ${ }^{25}$. Dessa forma, constata-se uma inconsistência na associação entre atividade física e dor lombar crônica na literatura científica, assim como uma ausência de explicação fisiológica, possivelmente em decorrência de um viés de causalidade reversa nessa associação ou de limitações inerentes aos estudos com delineamento transversal ${ }^{26}$. Neste estudo, evidenciou-se que $85,6 \%$ das mulheres investigadas eram fisicamente inativas, indicando uma possível falta de espaços públicos na cidade destinados à prática de atividade física. Assim, programas e ações de prevenção em saúde pública devem considerar esses achados e os aspectos relacionados ao gênero.

O estado nutricional, por sua vez, obtido por meio dos índices antropométricos, pode ser utilizado para predizer potenciais riscos à saúde. Entre os achados deste estudo, demonstrou-se que as mulheres com obesidade apresentaram uma probabilidade $25 \%$ maior de ter dor lombar, quando comparadas às mulheres com estado nutricional normal. Pesquisas prévias realizadas em diferentes populações também verificaram uma associação significativa entre obesidade e dor lombar, evidenciando que um maior IMC é um fator importante associado à maior ocorrência de dor lombar ${ }^{11,12,14,15,17}$. Nesse sentido, estudo conduzido com 3.182 adultos da cidade de Pelotas identificou uma tendência de aumento linear significativo na prevalência de dor lombar crônica equivalente ao aumento do $\mathrm{IMC}^{14}$. Segundo os autores, tal associação pode ser atribuída a uma provável "carga extra" que a estrutura osteomusculoarticular é obrigada a sustentar, alterando o equilíbrio biomecânico do corpo ${ }^{14}$. Além disso, um possível aumento do teor de gordura corporal na região dos músculos paravertebrais pode diminuir a mobilidade vertebral, alterando a nutrição do disco intervertebral e provocando a redução de sua altura ${ }^{12,17}$.

Quanto aos pontos fortes deste estudo, destacase a análise de uma amostra representativa e de base populacional, composta por mulheres com idade entre 
20 e 69 anos e residentes na zona urbana de São Leopoldo, Rio Grande do Sul, posto que, no Brasil, poucas pesquisas de base populacional investigaram a ocorrência de dor lombar e sua associação com possíveis determinantes em mulheres. Nesse sentido, este estudo explorou os potenciais fatores associados à ocorrência de dor lombar crônica em um grupo populacional vulnerável. Justifica-se assim a importância de realizar estudos sobre essa temática, especificamente com mulheres, pois a literatura científica vem apontando uma prevalência de dor lombar crônica significativamente maior nesse grupo populacional ${ }^{1,10-12,14,24}$. Essa diferença pode decorrer da dupla jornada de trabalho (labor/casa) a que as mulheres podem estar submetidas, ou de diferenças na sua estrutura anatômica corporal (densidade óssea, resistência muscular e estabilidade articular) em comparação aos homens ${ }^{13,14,26}$. Outro ponto positivo a ser ressaltado neste estudo diz respeito à utilização de instrumentos previamente validados para mensurar a ocorrência do desfecho de dor lombar crônica - ou seja, dor com no mínimo três meses de duração - e sua associação com diversas características sociodemográficas, comportamentais e nutricionais, mediante um modelo de análise multivariável. Ademais, para adequada identificação da região lombar, utilizou-se um cartão-resposta com uma ilustração da região corporal, ferramenta amplamente empregada em estudos prévios sobre a ocorrência de dor lombar crônica ${ }^{13,14,18,25}$.

Por outro lado, algumas limitações concernentes a este estudo devem ser mencionadas, como o fato de que a comparação entre estudos que utilizaram diferentes instrumentos para a avaliação da dor lombar pode ser limitada, já que a prevalência de dor lombar crônica nas mulheres investigadas nesta pesquisa, embora os fatores associados tenham sido semelhantes, mostrou-se mais elevada do que em outros estudos de base populacional. Além disso, por se tratar do relato de presença de dor nos últimos três meses, há a possibilidade de erro de recordatório e de viés da memória. Também é preciso levar em conta uma possível limitação na comparação entre estudos que exploraram a associação entre atividade física e dor lombar crônica, por não apresentarem uma padronização e por classificarem atividade física como uma questão dicotômica ou em diferentes níveis de intensidade. Além disso, como este estudo possui delineamento transversal, não se pode estabelecer relação causal entre os fatores explorados e o desfecho de dor lombar crônica, uma vez que as associações podem decorrer do viés de causalidade reversa. No entanto, os resultados deste trabalho podem subsidiar a formulação de novas hipóteses a serem testadas por meio de estudos longitudinais. Por fim, a intensidade da dor lombar não foi avaliada, impedindo a consideração desse aspecto para elaborar propostas de intervenção e prevenção da dor lombar na população investigada.

\section{CONCLUSÃO}

Este estudo identificou uma elevada prevalência de dor lombar crônica em mulheres, constatando como principais fatores associados a sua ocorrência a idade avançada, a inatividade física e a obesidade. Tendo em vista que a dor lombar crônica pode causar importantes limitações nas atividades da vida diária, é necessário conhecer seus fatores de risco relacionados aos hábitos de vida para que se possa estabelecer estratégias e políticas de promoção e prevenção em saúde.

\section{AGRADECIMENTOS}

Maria Teresa Anselmo Olinto recebeu bolsa de produtividade em pesquisa do $\mathrm{CNPq} / \mathrm{PQ}$ (processos $\mathrm{n}$. 307257/2013-4 e 307175/2017-0). Anderson Garcez recebeu bolsa de pós-doutorado júnior do $\mathrm{CNPq} / \mathrm{PDJ}$ (processo n. 161302/2019-0).

\section{REFERÊNCIAS}

1. Hoy D, Bain C, Williams G, March L, Brooks P, Blyth F, et al. A systematic review of the global prevalence of low back pain. Arthritis Rheum. 2012;64(6):2028-37. doi: 10.1002/ art.34347

2. Fransen M, Woodward M, Norton R, Coggan C, Dawe M, Sheridan N. Risk factors associated with the transition from acute to chronic occupational back pain. Spine (Phila Pa 1976). 2002;27(1):92-8. doi: 10.1097/00007632-200201010-00022

3. Ribeiro RP, Sedrez JA, Candotti CT, Vieira A. Relação entre a dor lombar crônica não específica com a incapacidade, a postura estática e a flexibilidade. Fisioter Pesqui. 2018;25(4):425-31. doi: 10.1590/1809-2950/18001925042018

4. Dionne CE, Dunn KM, Croft PR. Does back pain prevalence really decrease with increasing age? A systematic review. Age Ageing. 2006;35(3):229-34. doi: 10.1093/ageing/afj055

5. Last AR, Hulbert K. Chronic low back pain: evaluation and management. Am Fam Physician. 2009;79(12):1067-74.

6. Carey TS, Freburger J. Physical therapy for low back pain: what is it, and when do we offer it to patients? Ann Fam Med. 2014;12(2):99-101. doi: 10.1370/afm.1636

7. Freburger JK, Holmes GM, Agans RP, Jackman AM, Darter JD, Wallace AS, et al. The rising prevalence of chronic low 
back pain. Arch Intern Med. 2009;169(3):251-8. doi: 10.1001/ archinternmed.2008.543

8. Camargos MCS. Estimativas de expectativa de vida com doenças crônicas de coluna no Brasil. Ciênc Saúde Colet. 2014;19(6):1803-11. doi: 10.1590/1413-81232014196.15812013

9. Oliveira MM, Andrade SSCA, Souza CAV, Ponte JN, Szwarcwald CL, Malta DC. Problema crônico de coluna e diagnóstico de distúrbios osteomusculares relacionados ao trabalho (DORT) autorreferidos no Brasil: Pesquisa Nacional de Saúde, 2013. Epidemiol Serv Saúde. 2015;24(2):287-96. doi: 10.5123/ S1679-49742015000200011

10. Meucci RD, Fassa AG, Faria NMX. Prevalence of chronic low back pain: systematic review. Rev Saúde Pública. 2015;49. doi: 10.1590/S0034-8910.2015049005874

11. Garcia JBS, Hernandez-Castro JJ, Nunez RG, Pazos MA, Aguirre JO, Jreige A, et al. Prevalence of low back pain in Latin America: a systematic literature review. Pain Physician. 2014;17(5):379-91.

12. Gouveia N, Rodrigues A, Eusebio M, Ramiro S, Machado P, Canhao $\mathrm{H}$, et al. Prevalence and social burden of active chronic low back pain in the adult Portuguese population: results from a national survey. Rheumatol Int. 2016;36(2):183-97. doi: 10.1007/ s00296-015-3398-7

13. Matos MG, Hennington EA, Hoefel AL, Dias-da-Costa JS. Dor lombar em usuários de um plano de saúde: prevalência e fatores associados. Cad Saúde Pública. 2008;24(9):2115-22. doi: 10.1590/S0102-311X2008000900017

14. Silva MC, Fassa AG, Valle NCJ. Dor lombar crônica em uma população adulta do Sul do Brasil: prevalência e fatores associados. Cad Saúde Pública. 2004;20(2):377-85. doi: 10.1590/S0102-311X2004000200005

15. Bjorck-van Dijken C, Fjellman-Wiklund A, Hildingsson C. Low back pain, lifestyle factors and physical activity: a population based-study. J Rehabil Med. 2008;40(10):864-9. doi: 10.2340/16501977-0273

16. Santos LRCS, Assunção AA, Lima EP. Dor nas costas em adultos residentes em territórios quilombolas, Bahia. Rev Saúde Pública. 2014;48(5):750-7. doi: 10.1590/S0034-8910.2014048005317
17. Inoue G, Miyagi M, Uchida K, Ishikawa T, Kamoda H, Eguchi $Y$, et al. The prevalence and characteristics of low back pain among sitting workers in a Japanese manufacturing company. J Orthop Sci. 2015;20(1):23-30. doi: 10.1007/s00776-014-0644-x

18. Meucci RD, Fassa AG, Paniz VM, Silva MC, Wegman DH. Increase of chronic low back pain prevalence in a medium-sized city of southern Brazil. BMC Musculoskelet Disord. 2013;14:155. doi: 10.1186/1471-2474-14-155

19. Instituto Brasileiro de Geografia e Estatística. Censo Demográfico 2010. Rio de Janeiro: IBGE; 2010.

20. Chou R, Qaseem A, Snow V, Casey D, Cross JT Jr, Shekelle P, et al. Diagnosis and treatment of low back pain: a joint clinical practice guideline from the American College of Physicians and the American Pain Society. Ann Intern Med. 2007;147(7):478-91. doi: 10.7326/0003-4819-147-7-200710020-00006

21. Kuorinka I, Jonsson B, Kilbom A, Vinterberg H, Biering-Sorensen F, Andersson G, et al. Standardised Nordic questionnaires for the analysis of musculoskeletal symptoms. Appl Ergon. 1987;18(3):233-7. doi: 10.1016/0003-6870(87)90010-x

22. Victora CG, Huttly SR, Fuchs SC, Olinto MT. The role of conceptual frameworks in epidemiological analysis: a hierarchical approach. Int J Epidemiol. 1997;26(1):224-7. doi: 10.1093/ije/26.1.224

23. Almeida ICGB, Sa KN, Silva M, Baptista A, Matos MA, Lessa I. Prevalência de dor lombar crônica na população da cidade de Salvador. Rev Bras Ortop. 2008;43(3):96-102. doi: 10.1590/ S0102-36162008000200007

24. Nascimento PRC, Costa LOP. Prevalência da dor lombar no Brasil: uma revisão sistemática. Cad Saúde Pública. 2015;31(6):1141-56. doi: 10.1590/0102-311X00046114

25. Heneweer H, Vanhees L, Picavet HSJ. Physical activity and low back pain: a U-shaped relation? Pain. 2009;143(1-2):21-5. doi: 10.1016/j.pain.2008.12.033

26. Zanuto EAC, Codogno JS, Christofaro DGD, Vanderlei LCM, Cardoso JR, Fernandes RA. Prevalence of low back pain and associated factors in adults from a middle-size Brazilian city. Ciên Saúde Colet. 2015;20(5):1575-82. doi: 10.1590/1413-81232015205.02162014 